\title{
Ultrasound guidance and risk
} for intravascular catheter-related infections among peripheral arterial catheters: a post-hoc analysis of two large randomized-controlled trials

\author{
Niccolò Buetti ${ }^{*} \mathbb{B}$, Stéphane Ruckly' ${ }^{1}$, Jean-Christophe Lucet ${ }^{1,2}$, Lila Bouadma ${ }^{1,3}$, Carole Schwebel ${ }^{4,8}$,
} Olivier Mimoz ${ }^{5,6,7}$ and Jean-François Timsit ${ }^{1,3}$

\begin{abstract}
Background: The impact on infectious risk of ultrasound guidance at insertion remains controversial in short-term arterial catheters (ACs). The present study investigated the association between ultrasound guidance (US) during AC insertion and major catheter-related infections (MCRI), catheter-related bloodstream infections (CR-BSI) or colonization, using univariate and multivariate marginal Cox model for clustered data. The skin colonization at catheter removal was evaluated to explain our results.

Results: We used individual data from two multicenter randomized-controlled trials (RCTs) that included a total of 3029 patients, 10 ICUs and 3950 ACs. US guidance was used for 386 (9.8\%) catheter placements. In the univariate Cox model analysis, AC insertion with US versus without US exhibited similar risks for MCRI (HR 0.86, Cl 95\% 0.27-2.72, $p=0.79$ ), CR-BSI (HR 0.87, Cl 95\% 0.20-3.72, $p=0.85$ ) and catheter colonization (HR 1.31, Cl 95\% 0.92-1.86, $p=0.13$ ). After adjustment on confounders, risks associated with US guidance remained similar versus non-US for MCRI (HR $0.71, \mathrm{Cl} 95 \% 0.23-2.24, p=0.56$ ), CR-BSI (HR 0.71, Cl 95\% 0.17-3.00, $p=0.63$ ) and catheter colonization (HR 0.92, Cl $95 \% 0.63-1.34, p=0.67)$. No differences between US and non-US for MCRI, CR-BSI and colonization were observed according to the insertion site, radial or femoral. At catheter removal, the skin colonization was similar between US and non-US groups $(p=0.69)$.
\end{abstract}

Conclusions: Using the largest dataset ever collected from large multi-centric RCTs conducted with relatively consistent insertion and maintenance catheter protocols, we showed that the risk of infectious complications for ACs inserted under US guidance is not superior compared to those inserted without US guidance.

Trial registration These studies were registered within ClinicalTrials.gov (numbers NCT01629550 and NCT 01189682).

Keywords: Catheter, Intravascular, Arterial catheter, Catheter tip, Ultrasound, Ultrasound guidance, Arterial, Catheterrelated bloodstream infection, infectious risk

*Correspondence: niccolo.buetti@gmail.com

1 University of Paris, INSERM IAME, U1137, Team DeSCID, Paris, France

Full list of author information is available at the end of the article

\section{Background}

Arterial catheters (ACs) are instrumental for managing critically ill patients, to facilitate hemodynamic monitoring and frequent blood sampling [1]. To date, the duration of catheter maintenance of ACs is similar to that 
of central venous catheters, thus now associated with a substantial infection risk [2]. Traditionally, anatomical 'landmarks' on the body surface were used to find the correct place in which to insert catheters. However, arterial catheterization using anatomical 'landmarks' is associated with an increase in the number of attempts and time needed for successful cannulation [3-5]. The current literature shows that ultrasound imaging may offer gains in safety and quality compared with an anatomical landmark technique. However, the ultrasound guidance effect on infectious risk remains controversial. Indeed, the ultrasound may have its own infectious risk. To our knowledge, no large randomized-controlled trials (RCTs) analyzed the infectious risk between both $\mathrm{AC}$ insertion strategies, anatomical landmarks' technique and ultrasound guidance. Our primary aim was to investigate the association between ultrasound guidance (US) for AC insertion and the intravascular catheter-related infection or colonization, using data gathered for two large RCTs with an extensive prospective data collection at catheter insertion and catheter removal $[6,7]$.

\section{Methods \\ Design}

We used the data from two large RCTs that investigated various prevention strategies, and for which an extensive prospective data collection at catheter insertion and catheter removal was performed $[6,7]$.

\section{Patients and setting}

Patients were recruited from 2011 to 2014 in various intensive care units (ICUs) in France as soon as they required a catheterization with a short-term central venous catheter (CVC) or a peripheral AC with an expected duration of use of more than $48 \mathrm{~h}$.

\section{Catheters}

For the current study we included only data related to AC. All study centers complied with the French recommendations for catheter insertion and care, which are similar to CDC recommendations [8]: (1) maximal sterile barrier precautions (large sterile drape; surgical hand antisepsis; and mask, cap, sterile gloves, and gown); (2) the site of insertion was left to the discretion of the physician caring for the patient; (3) alcoholic povidone iodine solution or chlorhexidine gluconate was used for skin antisepsis at catheter insertion and during dressing changes; (4) semipermeable chlorhexidine-impregnated or standard dressing was used at all insertion sites and was changed $24 \mathrm{~h}$ after catheter insertion and then every 3 or 7 days according to standard practice in each ICU. Leaking, soiled, or wet dressings were changed immediately. Ultrasound guidance was used at the discretion of the attending physician and this variable was routinely collected. A transducer with a sterile sheath was used to perform vascular access procedures. Sterile gel was used. Antiseptic- or antibiotic-impregnated ACs were not used in any of the study ICUs. A check list was routinely used. The catheters were removed if unnecessary or if an infection was suspected. The patients underwent follow-up until $48 \mathrm{~h}$ after ICU discharge.

\section{Definitions and outcomes}

According to French and American guidelines, the following definitions were used $[9,10]$. Catheter colonization was defined as a quantitative catheter tip culture yielding $\geq 1000$ colony-forming units $(\mathrm{cfu}) / \mathrm{mL}$. A catheter-related clinical sepsis without bloodstream infection (BSI) was a combination of body temperature $\left(\geq 38.5^{\circ} \mathrm{C}\right.$ or $\leq 36.5^{\circ} \mathrm{C}$ ); catheter colonization; presence of pus at the insertion site or resolution of clinical sepsis after catheter removal; and the absence of any other infectious focus. A catheter-related bloodstream infection (CR-BSI) was a combination of (1) one or more positive peripheral blood cultures sampled $48 \mathrm{~h}$ before or after catheter removal; (2) the isolation of the same organism from the colonized catheter or from the catheter insertion site, or a blood culture differential time to positivity of $2 \mathrm{~h}$ or more [11]; and (3) no apparent source of bacteremia other than the catheter. If a patient had a positive blood culture for coagulase-negative staphylococci (CoNS), the same pulsotype from the strains recovered from the catheter and blood culture was required for a diagnosis of CR-BSI. A major catheter-related infection (MCRI) was defined as either a catheter-related clinical sepsis without BSI, or a CR-BSI. For patients without any catheter cultures, a blinded adjudication committee determined whether a MCRI was present; sepsis or BSI were classified as catheter-related when there was no other detectable cause of sepsis with or without BSI. The skin colonization was evaluated using semi-quantitative insertion-site cultures: the insertion site was sampled immediately before catheter removal. Because the size of the counting surface was different across studies, we created a semi-quantitative variable with sterile (i.e., negative quantitative cultures), low-grade skin colonization, and high-grade skin colonization according to the median of quantitative cultures obtained in each study.

\section{Statistical analysis}

Characteristics of patients and catheters were described as count (percent) or median (interquartile range) for qualitative and quantitative variables, respectively, and were compared between catheters groups using Chi square, Fisher or Mann-Whitney tests, as appropriate. 
The statistical plan had two objectives: (1) to identify the risk differences in MCRI, CR-BSI and catheter colonization between catheters inserted using US guidance and catheter inserted with using anatomical 'landmarks' (non-US); (2) to perform a confirmatory analysis analyzing differences in skin colonization at removal between US and non-US.

For the first objective, we used a marginal Cox model for clustered data (PROC PHREG of SAS), to take into account a possible clustering effect of multiple catheters per patient. This model takes into account the censored nature of the data and possible intra-cluster dependence using a robust sandwich covariate estimate. Analyses were stratified by ICU and data were censored at 28 days since catheter insertion. Hazard risk for MCRI, CR-BSI and catheter colonization was evaluated by univariate and multivariate analysis. The variable "ultrasound guidance" (US vs. non-US) was forced in our multivariate models and the other variables showing significance in the univariate analysis were used as adjustment factors. The choice of adjustment variables was based on the results of the univariate analysis and refined by including clinically relevant variables (i.e., Simplified Acute Physiology Score [SAPS] II score). The proportionality of hazard risks for catheter type was tested using Martingale residuals.

Confirmatory subgroup analyses were performed according to the insertion site (radial and femoral) and the duration of catheter maintenance $(\leq 7$ days and $>7$ days). Tests were two-tailed, with $p<0.05$ being considered significant. All analyses were performed using SAS (version 9.4; SAS Institute, Cary, NC). All studies were approved by the national ethics committee.

\section{Results}

Patients and catheters

A total of 3029 patients were included by 10 ICUs in this study and 3950 ACs were analyzed (Fig. 1).

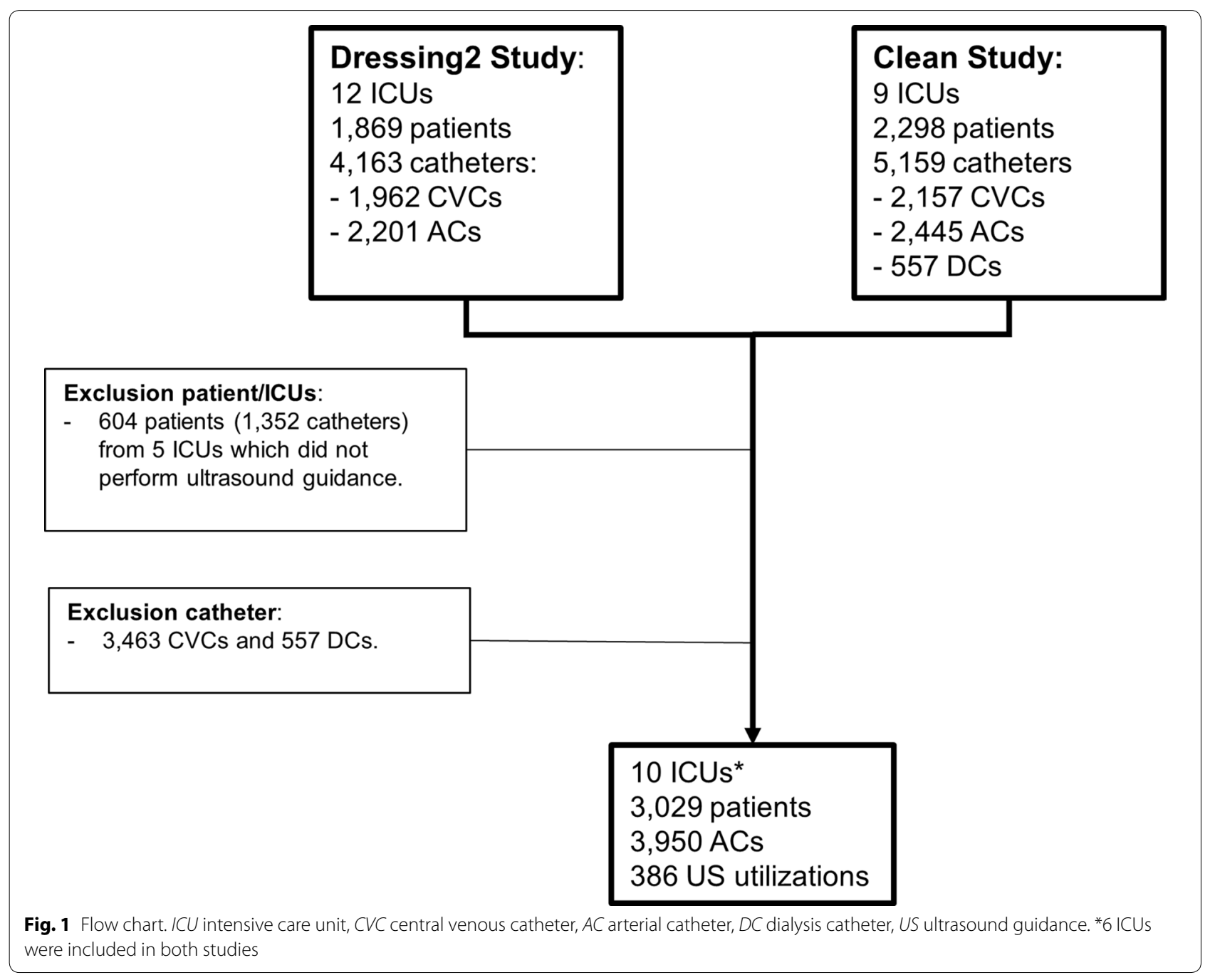


The US was used for 386 catheter placements (356 different patients). Characteristics of the patients and catheters are described in Tables 1 and 2. In the US group, the patients were more frequently admitted for shock (48\%) and their SAPS II at study inclusion was significantly higher $(54$, IQR $[41 ; 68])$ than that of patients without US.

US was more frequently used by junior operators $(70 \%)$ and for the femoral site insertion (59\%). In addition, the skin antisepsis and the dressing used were different according to the ultrasound utilization. We observed 32 MCRIs, 21 CR-BSI and 307 colonizations.

\section{Catheter infections and colonizations}

In the univariate Cox model analysis, the risk for MCRI (HR 0.86, CI 95\% 0.27-2.72, $p=0.79$ ), CR-BSI (HR 0.87, CI 95\% 0.20-3.72, $p=0.85$ ) and catheter colonization (HR 1.31, CI 95\% 0.92-1.86, $p=0.13$ ) was similar for ACs in the US group compared to those of the non-US group (Additional file 1: Tables S1-S3). The proportionality of hazard was respected for MCRI, CR-BSI and colonization.

In multivariate marginal Cox model, US showed similar risk compared to non-US for MCRI (HR 0.71, CI 95\% $0.23-2.24, p=0.56$ ), CR-BSI (HR 0.71, CI 95\% 0.17-3.00, $p=0.63$ ) and catheter colonization (HR 0.92, CI 95\%

Table 1 Patients' characteristics

\begin{tabular}{lllr}
\hline & $\begin{array}{l}\text { Non-US } \\
(\boldsymbol{n}=\mathbf{2 6 7 3})\end{array}$ & US $(\boldsymbol{n = 3 5 6 )}$ & $\boldsymbol{p}$ value \\
\hline Sex & & & \\
$\quad$ Female & $934(34.9)$ & $137(38.5)$ & 0.19 \\
$\quad$ Male & $1739(65.1)$ & $219(61.5)$ & \\
Age, median (IQR) & $63[52 ; 74]$ & $63[52.5 ; 73]$ & 0.98 \\
Reason for ICU admission & & & \\
Renal failure & $113(4.2)$ & $17(4.8)$ & $<0.01$ \\
Shock & $920(34.4)$ & $171(48)$ & \\
Coma & $265(9.9)$ & $19(5.3)$ & \\
Other & $362(13.5)$ & $46(12.9)$ & \\
Respiratory failure & $811(30.3)$ & $78(21.9)$ & \\
Trauma & $202(7.6)$ & $25(7)$ & \\
No comorbidity & $1803(67.5)$ & $242(68)$ & 0.84 \\
Chronic renal failure & $101(3.8)$ & $15(4.2)$ & 0.69 \\
Chronic cardiac failure & $154(5.8)$ & $24(6.7)$ & 0.46 \\
Diabetes mellitus & $175(6.5)$ & $21(5.9)$ & 0.64 \\
Chronic respiratory failure & $153(5.7)$ & $11(3.1)$ & 0.04 \\
Immunosuppression & $234(8.8)$ & $36(10.1)$ & 0.40 \\
Hematologic neoplasia & $119(4.5)$ & $13(3.7)$ & 0.49 \\
MV at admission & $1912(71.5)$ & $268(75.3)$ & 0.14 \\
Vasopressor at admission & $1067(39.9)$ & $99(27.8)$ & $<0.01$ \\
SAPS II score, median (IQR) & $50[37 ; 65]$ & $54[41 ; 68]$ & 0.02 \\
\hline OR & & \\
\end{tabular}

$I Q R$ interquartile range, ICU intensive care unit, $M V$ mechanical ventilation, SAPS II score simplified Acute Physiology Score II. In 30 patients, the ultrasound guidance was used $>1$ time
Table 2 Catheters' characteristics

\begin{tabular}{lllc}
\hline & $\begin{array}{l}\text { Non-US } \\
(\mathbf{n}=\mathbf{3 5 6 4})\end{array}$ & US (n=386) & p-value \\
\hline $\begin{array}{l}\text { Catheter days, median (IQR) } \\
\text { Experience of the operator }\end{array}$ & $5[2,9]$ & $5[2,9]$ & 0.65 \\
$\begin{array}{l}<50 \text { procedures } \\
\geq 50 \text { procedures }\end{array}$ & $2131(59.8)$ & $269(69.7)$ & $<0.01$ \\
Insertion site & $1433(40.2)$ & $117(30.3)$ & \\
Femoral & $1081(30.3)$ & $226(58.5)$ & $<0.01$ \\
Radial & $2483(69.7)$ & $160(41.5)$ & \\
Dressing & & & \\
CHG-impregnated & $763(21.4)$ & $11(2.8)$ & $<0.01$ \\
Standard dressing & $2801(78.6)$ & $375(97.2)$ & \\
Skin antisepsis & & & \\
Not CHG & $1482(41.6)$ & $188(48.7)$ & $<0.01$ \\
CHG & $2082(58.4)$ & $198(51.3)$ & \\
MV at insertion & $2467(69.2)$ & $280(72.5)$ & 0.18 \\
Vasopressor at insertion & $1471(41.3)$ & $223(57.8)$ & $<0.01$ \\
Antibiotic at insertion & $1933(54.2)$ & $235(60.9)$ & 0.01 \\
MCRI & $29(0.8)$ & $3(0.8)$ & 0.94 \\
CR-BSI & $19(0.5)$ & $2(0.5)$ & 0.97 \\
Colonization & $269(7.5)$ & $38(9.8)$ & 0.11 \\
\hline
\end{tabular}

IQR interquartile range, ICU intensive care unit, $M V$ mechanical ventilation, SAPS II score Simplified Acute Physiology Score II, CHG chlorhexidine gluconate, MCRI major catheter-related infection, $C R-B S /$ catheter-related bloodstream infection

0.63-1.34, $p=0.67$, Fig. 2 and Additional file 1: Tables S1-S3).

Variables independently associated with MCRI (Additional file 1: Table S1) were non-chlorhexidine skin antisepsis (HR 6.42, 95\% CI 2.58-15.98, $p<0.01$ ) and vasopressor at insertion (HR $0.49,95 \%$ CI $0.23-1.05$, $p=0.07)$. Variables independently associated with CRBSI (Additional file 1: Table S2) were non-chlorhexidine skin antisepsis (HR 6.07, 95\% CI 2.2-16.74, $p<0.01$ ) and antibiotics at insertion (HR 0.34, 95\% CI 0.13-0.87, $p=0.02$ ). Variables independently associated with colonization (Additional file 1: Table S3) were vasopressor at admission (HR $0.78,95 \%$ CI $0.60-0.99, p=0.05$ ), femoral insertion site (HR 1.50; 95\% CI 1.18-1.92, $p<0.01$ ), standard dressing (HR 2.53, 95\% CI 1.59-4.03, $p<0.01$ ), non-chlorhexidine skin disinfection (HR 6.0, 95\% CI 4.49-8.01, $p<0.01$ ), mechanical ventilation at insertion (HR 0.80, 95\% CI 0.62-1.03, $p=0.08$ ) and antibiotics at insertion (HR 0.56, 95\% CI 0.44-0.70, $p<0.01$ ).

\section{Confirmatory analyses}

Among femoral catheters $(n=1307)$, no differences between US and non-US for MCRI, CR-BSI and colonization were observed (Fig. 2). Similarly, for radial catheters (=2643) a similar risk for MCRI, CR-BSI and colonization was showed. In the subgroup analysis including 


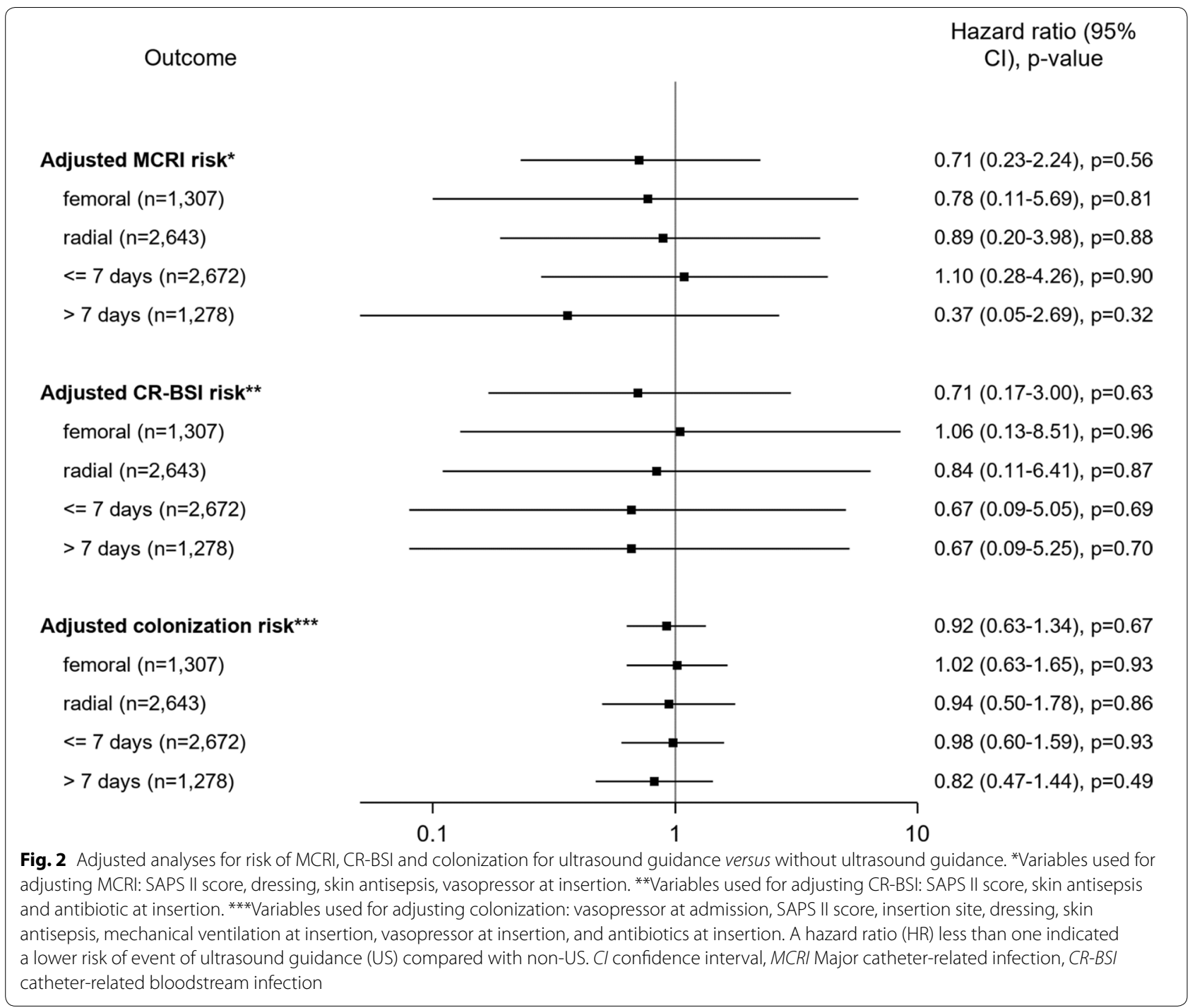

only catheters with $\leq 7$ days maintenance $(n=2672)$, the MCRI, CR-BSI and colonization risk for US did not differ from non-US.

The skin colonization at catheter removal was similar between US and non-US groups ( $p=0.69$, Table 3$)$. No difference was observed in the different subgroups.

\section{Discussion}

Using prospectively collected data from two RCTs, we showed that the US at AC insertion did not influence the risk of intravascular catheter infections. Data in the literature about the role of US in intravascular AC infections are scarce. The current literature mostly focus only on first-attempt failure, mean attempts to success, mean time to success, and the occurrence of hematoma or venipuncture complications [3-5]. Interestingly, all RCTs investigating the role of US for the radial artery in adults assessed only non-infectious complications, thus disregarding intravascular catheter infections [12-17]. Similarly, among femoral artery cannulations, all RCTs were performed in cardiologic patients and mainly focused on short-term complications without considering catheter infections [18-21]. To date, the role of US in the context of intravascular arterial catheter infection is not clear. The use of an additional device, such as ultrasound, may complicate $\mathrm{AC}$ insertion and set the stage for breaches in aseptic non-touch technique, and the gel used for optimizing visibility may increase the risk of catheter infection $[22,23]$. However, a shorter insertion time and fewer insertion attempts may counterbalance the risk for infection. In an environment of consistent catheter care representing the largest dataset ever assembled, we showed that the US did not increase the infectious risk among 
Table 3 Skin colonization at catheter removal (main group and subgroup analyses)

\begin{tabular}{llll}
\hline & No US & US & p value* \\
\hline All & & & \\
High-grade colonization & $886(34.5)$ & $108(36.9)$ & 0.69 \\
Low-grade colonization & $865(33.6)$ & $93(31.7)$ & \\
$\begin{array}{l}\text { Sterile } \\
\text { Femoral }\end{array}$ & $820(31.9)$ & $92(31.4)$ & \\
High-grade colonization & $268(37.1)$ & $57(35.4)$ & 0.84 \\
Low-grade colonization & $243(33.6)$ & $58(36)$ & \\
Sterile & $212(29.3)$ & $46(28.6)$ & \\
Radial & & & \\
$\quad$ High-grade colonization & $618(33.4)$ & $51(38.6)$ & 0.22 \\
Low-grade colonization & $622(33.7)$ & $35(26.5)$ & \\
Sterile & $608(32.9)$ & $46(34.8)$ & \\
>7 days & & & \\
High-grade colonization & $506(29.2)$ & $69(34.5)$ & 0.30 \\
Low-grade colonization & $624(36)$ & $66(33)$ & \\
Sterile & $601(34.7)$ & $65(32.5)$ & \\
$>7$ days & & & \\
High-grade colonization & $380(45.2)$ & $39(41.9)$ & 0.78 \\
Low-grade colonization & $241(28.7)$ & $27(29)$ & \\
Sterile & $219(26.1)$ & $27(29)$ & \\
\hline
\end{tabular}

Skin cultures at removal were not performed for 1086 catheters. ${ }^{*}$ Chi square test was performed

ACs. No differences were observed according to the body site of insertion, femoral or radial, and the skin colonization at removal was similar between the two groups. Therefore, our results support the growing evidence that recommends using US routinely $[1,3-5]$.

Our study has several limitations. First, its design is observational and the US utilization was not randomized. However, we presented exhaustive data that have been prospectively collected by trained investigators and study monitors during RCTs, and we adjusted our analyses on major confounders. Second, no data on the US type (e.g., Doppler vs. ultrasound guidance) or sterile sheath used were included. Third, all RCTs were conducted in University-affiliated ICUs in France from 2011 to 2014, thus limiting the generalizability of the results. However, aseptic insertion techniques have not been changed since 2014. Fourth, a post-hoc analysis of our dataset showed a $22.5 \%$ probability that US was associated with a falsely significant increased HR of $\geq 1.1$ for MCRI $(18 \%$ for colonization). Fifth, no information on the number of attempts and other mechanical complications were included. However, these outcomes were exhaustively explored in previous analyses. Sixth, catheter cultures were not performed in 406 catheters. However, the rate of missing information was not different between both groups. Finally, we described a large database designed to investigate the impact of certain prevention measures, and interactions may have occurred among the various study groups. However, our statistical analyses considered these potential drawbacks.

\section{Conclusion}

Using the largest dataset ever collected from large multicentric RCTs conducted with relatively consistent insertion and maintenance catheter protocols, we showed that the risk of infectious complications for arterial catheters inserted under US guidance is not superior compared to those inserted without US guidance. Large RCTs primarily designed to investigate the true impact of US guidance on the infectious risk of arterial catheters are warranted.

\section{Supplementary information}

Supplementary information accompanies this paper at https://doi. org/10.1186/s13613-020-00705-4.

Additional file 1. Univariate and multivariate Cox models for MCRI, CR-BSI and colonization.

\section{Abbreviations}

AC: Arterial catheter; BSI: Bloodstream infection; Cl: Confidence interval; CVC: Central venous catheter; CR-BSI: Catheter-related Bloodstream Infection; CoNS: Coagulase-negative staphylococci; HR: Hazard ratio; ICU: Intensive Care Unit; MCRI: Major Catheter-related Infection; RCT: Randomized-controlled trial; SAPS: Simplified Acute Physiology Score; US: Ultrasound guidance.

\section{Acknowledgements \\ The authors thank Céline Féger, M.D., (EMIBiotech) for her editorial sup- port and the Swiss National Science Foundation and the Bangerter Rhyner Foundation.}

\section{Authors' contributions}

NB, SR and JFT analyzed and interpreted the data. JFT, JCL and OM were responsible for the data collection of the two RCTs. NB and JFT were the major contributors in writing the manuscript. All authors read and approved the final manuscript.

\section{Funding}

NB is currently receiving a Mobility grant from the Swiss National Science Foundation (Grant Number: P400PM_183865) and a grant from the BangerterRhyner Foundation. These grants support his fellowship in France.

\section{Availability of data and materials}

The datasets used and/or analyzed during the current study are available from the corresponding author upon reasonable request.

Ethics approval and consent to participate

All studies were approved by the national ethics committee.

\section{Consent for publication}

Not applicable.

\section{Competing interests}

The authors declare that they have no competing interests. JFT received fees for lectures to $3 \mathrm{M}, \mathrm{MSD}$, Pfizer, and Biomerieux. JFT received research grants from Astellas, 3 M, MSD, and Pfizer. JFT participated to advisory boards of $3 \mathrm{M}$, MSD, Bayer Pharma, Nabriva, and Pfizer. JCL received fees for lectures for $3 \mathrm{M}$, 
Pfizer MSD, and research grants from Anios. OM received fees for lectures for $3 \mathrm{M}$ and $\mathrm{BD}$. OM received research grants from $\mathrm{BD}$.

\section{Author details}

${ }^{1}$ University of Paris, INSERM IAME, U1 137, Team DeSCID, Paris, France. ${ }^{2}$ AP-HP, Infection Control Unit, Bichat- Claude Bernard University Hospital, 46 Rue Henri Huchard, 75877 Paris Cedex, France. ${ }^{3}$ Medical and Infectious Diseases Intensive Care Unit, AP-HP, Bichat-Claude Bernard University Hospital, 46 Rue Henri Huchard, 75877 Paris Cedex, France. ${ }^{4}$ Medical Intensive Care Unit, Grenoble University Hospital, Grenoble 1 University, La Tronche, France. ${ }^{5}$ Services Des Urgences Adultes and SAMU 86, Centre Hospitalier Universitaire de Poitiers, 86021 Poitiers, France. ${ }^{6}$ Université de Poitiers, Poitiers, France. ${ }^{7}$ Inserm U1070, Poitiers, France. ${ }^{8}$ INSERM UMR S 1039, Radiopharmaceutiques Biocliniques, Faculté de Médecine de Grenoble, Domaine de la Merci, 38700 La Tronche, France.

Received: 24 February 2020 Accepted: 26 June 2020

Published online: 08 July 2020

\section{References}

1. Wang A, Hendin A, Millington SJ, Koenig S, Eisen LA, Shiloh AL. Better with ultrasound: arterial line placement. Chest. 2020;157(3):574-9.

2. Lucet JC, Bouadma L, Zahar JR, Schwebel C, Geffroy A, Pease S, Herault MC, Haouache H, Adrie C, Thuong M, et al. Infectious risk associated with arterial catheters compared with central venous catheters. Crit Care Med. 2010;38(4):1030-5.

3. White L, Halpin A, Turner M, Wallace L. Ultrasound-guided radial artery cannulation in adult and paediatric populations: a systematic review and meta-analysis. Br J Anaesth. 2016;116(5):610-7.

4. Gu WJ, Wu XD, Wang F, Ma ZL, Gu XP. Ultrasound guidance facilitates radial artery catheterization: a meta-analysis with trial sequential analysis of randomized controlled trials. Chest. 2016;149(1):166-79.

5. Sobolev M, Slovut DP, Lee Chang A, Shiloh AL, Eisen LA. Ultrasoundguided catheterization of the femoral artery: a systematic review and meta-analysis of randomized controlled trials. J Invas Cardiol. 2015;27(7):318-23.

6. Timsit JF, Mimoz O, Mourvillier B, Souweine B, Garrouste-Orgeas M, Alfandari S, Plantefeve G, Bronchard R, Troche G, Gauzit R, et al. Randomized controlled trial of chlorhexidine dressing and highly adhesive dressing for preventing catheter-related infections in critically ill adults. Am J Respir Crit Care Med. 2012;186(12):1272-8.

7. Mimoz O, Lucet JC, Kerforne T, Pascal J, Souweine B, Goudet V, Mercat A, Bouadma L, Lasocki S, Alfandari S, et al. Skin antisepsis with chlorhexidine-alcohol versus povidone iodine-alcohol, with and without skin scrubbing, for prevention of intravascular-catheter-related infection (CLEAN): an open-label, multicentre, randomised, controlled, two-by-two factorial trial. Lancet. 2015;386(10008):2069-77.

8. O'Grady NP, Alexander M, Burns LA, Dellinger EP, Garland J, Heard SO, Lipsett PA, Masur H, Mermel LA, Pearson ML, et al. Guidelines for the prevention of intravascular catheter-related infections. Clin Infect Dis. 2011;52(9):e162-93.

9. Timsit JF. Updating of the 12th consensus conference of the Societe de Reanimation de langue francaise (SRLF): catheter related infections in the intensive care unit. Ann Fr Anesth Reanim. 2005;24(3):315-22.

10. Mermel LA, Allon M, Bouza E, Craven DE, Flynn P, O'Grady NP, Raad II, Rijnders BJ, Sherertz RJ, Warren DK. Clinical practice guidelines for the diagnosis and management of intravascular catheter-related infection: 2009 Update by the Infectious Diseases Society of America. Clin Infect Dis. 2009;49(1):1-45.

11. Blot F, Nitenberg G, Chachaty E, Raynard B, Germann N, Antoun S, Laplanche A, Brun-Buisson C, Tancrede C. Diagnosis of catheter-related bacteraemia: a prospective comparison of the time to positivity of hubblood versus peripheral-blood cultures. Lancet. 1999;354(9184):1071-7.

12. Levin $P D$, Sheinin $O$, Gozal Y. Use of ultrasound guidance in the insertion of radial artery catheters. Crit Care Med. 2003;31(2):481-4.

13. Shiver S, Blaivas M, Lyon M. A prospective comparison of ultrasoundguided and blindly placed radial arterial catheters. Acad Emerg Med. 2006;13(12):1275-9.

14. Hansen MA, Juhl-Olsen P, Thorn S, Frederiksen CA, Sloth E. Ultrasonography-guided radial artery catheterization is superior compared with the traditional palpation technique: a prospective, randomized, blinded, crossover study. Acta Anaesthesiol Scand. 2014;58(4):446-52.

15. Peters C, Schwarz SK, Yarnold CH, Kojic K, Kojic S, Head SJ. Ultrasound guidance versus direct palpation for radial artery catheterization by expert operators: a randomized trial among Canadian cardiac anesthesiologists. Can J Anaesth. 2015:62(11):1161-8.

16. Seto AH, Roberts JS, Abu-Fadel MS, Czak SJ, Latif F, Jain SP, Raza JA, Mangla A, Panagopoulos G, Patel PM, et al. Real-time ultrasound guidance facilitates transradial access: RAUST (Radial Artery access with Ultrasound Trial). JACC Cardiovasc Interv. 2015;8(2):283-91.

17. Ueda K, Bayman EO, Johnson C, Odum NJ, Lee JJ. A randomised controlled trial of radial artery cannulation guided by Doppler vs. palpation vs. ultrasound. Anaesthesia. 2015;70(9):1039-44.

18. Dudeck O, Teichgraeber U, Podrabsky P, Lopez Haenninen E, Soerensen $\mathrm{R}$, Ricke J. A randomized trial assessing the value of ultrasound-guided puncture of the femoral artery for interventional investigations. Int J Cardiovasc Imaging. 2004;20(5):363-8.

19. Gedikoglu M, Oguzkurt L, Gur S, Andic C, Sariturk C, Ozkan U. Comparison of ultrasound guidance with the traditional palpation and fluoroscopy method for the common femoral artery puncture. Catheter Cardiovasc Interv. 2013;82(7):1187-92.

20. Slattery MM, Goh GS, Power S, Given MF, McGrath FP, Lee MJ. Comparison of ultrasound-guided and fluoroscopy-assisted antegrade common femoral artery puncture techniques. Cardiovasc Intervent Radiol. 2015;38(3):579-82.

21. Seto AH, Abu-Fadel MS, Sparling JM, Zacharias SJ, Daly TS, Harrison AT, Suh WM, Vera JA, Aston CE, Winters RJ, et al. Real-time ultrasound guidance facilitates femoral arterial access and reduces vascular complications: FAUST (Femoral Arterial Access With Ultrasound Trial). JACC Cardiovasc Interv. 2010;3(7):751-8.

22. Chittick P, Russo V, Sims M, Robinson-Dunn B, Oleszkowicz S, Sawarynski K, Powell K, Makin J, Darnell E, Boura JA, et al. An outbreak of Pseudomonas aeruginosa respiratory tract infections associated with intrinsically contaminated ultrasound transmission gel. Infect Control Hosp Epidemiol. 2013;34(8):850-3.

23. Abdelfattah R, Al-Jumaah S, Al-Qahtani A, Al-Thawadi S, Barron I, AlMofada S. Outbreak of Burkholderia cepacia bacteraemia in a tertiary care centre due to contaminated ultrasound probe gel. J Hosp Infect. 2018;98(3):289-94.

\section{Publisher's Note}

Springer Nature remains neutral with regard to jurisdictional claims in published maps and institutional affiliations. 